\title{
On the Wall-Modeled LES use of ZDES in compressible flows
}

\author{
Nicolas Renard*, Sébastien Deck ${ }^{\dagger}$ and Pierre-Élie Weiss ${ }^{\ddagger}$ \\ ONERA The French Aerospace Lab, Meudon, France
}

\begin{abstract}
The present study is devoted to the use of the Zonal Detached Eddy Simulation (mode 3) hybrid RANS/LES method as a Wall-Modeled Large Eddy Simulation technique in compressible flows. The first section is devoted to a supersonic turbulent boundary layer test case, showing that the performance of ZDES mode 3 is satisfying in supersonic conditions, validating this WMLES method previously analyzed at low Mach numbers. Then the interaction of this boundary layer with an oblique shock wave is considered, with a promising qualitative agreement with experimental data and enhanced predictions compared with RANS. Finally, a new strategy by Deck et al. [1] for a low-noise resolved turbulence generation is presented, which is expected to be instrumental for the next use of ZDES mode 3 in aeroacoustic studies.
\end{abstract}

\section{Introduction}

The increasing demand for high-fidelity computational aerodynamics motivates more and more frequently unsteady turbulence modelling. Among the possible approaches, the hybrid RANS/LES paradigm is suited for the industrial needs since it combines a high degree of resolution in the zones of interest, which are treated in LES, with a cost-efficient RANS treatment of the other regions of the flow. The Zonal Detached Eddy Simulation technique (ZDES) [2] developed since 2002 enables a flexible definition of the zones of interest and has been widely validated and used for aerospace engineering applications (see e.g. [3]) as well as for academic research. The ZDES approach features two modes for massively separated flows and a third mode for the resolution of wall-bounded turbulence. Resolving turbulent fluctuations in attached boundary layers may be required to reach a specified level of description of the unsteady flow (e.g. for unsteady loading or aeroacoustic predictions), to enhance universality compared with RANS models (e.g. with respect to adverse pressure gradient effects) or to include upstream history effects associated with the boundary layer unsteady dynamics (e.g. mild flow separation, shock wave / boundary layer interaction). The cost of resolving the inner layer dynamics of attached boundary layers is however prohibitive for industrial applications, so that wall modelling is mandatory. In ZDES mode 3, a near-wall RANS zone is defined and performs as a wall model for the LES of the outer layer, as detailed in [4-6]. This WMLES strategy relies on the hybrid RANS/LES framework, like other methods such as the WMLES branch of IDDES [7] or the LES/RANS blending by Choi, Edwards \& Baurle [8].

More specifically, in ZDES mode 3 the near-wall RANS / outer LES interface is treated in a passive way by simply switching the length scale used in the Spalart-Allmaras turbulence model [9] from the wall distance $d_{w}$ in the RANS zone to the cell size estimate (based on the cell volume) in the LES zone (i.e. $\tilde{d}=d_{w}$ if $d_{w}<d_{w}^{\text {interface }}, \min \left(d_{w}, C_{\mathrm{DES}} \Delta_{\mathrm{vol}}\right)$ otherwise). This simple treatment is robust and does not introduce empiricism or spurious noise, but the height of the RANS/LES interface must be carefully set, independent from the mesh resolution [4] (which means there is no meshing constraint, unlike other methods) and at an outer-scaled position, for instance $d_{w}^{\text {interface }} / \delta=0.1$ [5] (which requires preprocessing). Further refinement is proposed in [6] but not crucial here.

The investigation and validation of ZDES mode 3 [4, 6, 10] and of the turbulent inflow conditions required for such WMLES [1, 11, 12] has been mainly focused on subsonic boundary layers at high Reynolds numbers. Compressibility effects that might affect ZDES mode 3, for instance in applications at higher Mach numbers or in aeroacoustic studies, have been addressed in a limited number of studies so far. The present paper investigates compressibility effects in ZDES mode 3 in three steps. First, simulations of a supersonic turbulent boundary layer are presented. The interaction of the resolved turbulent boundary layer with a shock wave is then considered. Finally, the new strategy by Deck et al. [1] to reduce the spurious acoustic footprint of the resolved turbulence generation needed for a WMLES is discussed.

*Research Scientist, Aerodynamics, Aeroelasticity, Acoustics Department, Advanced Turbulence Modelling \& Simulation Unit, nicolas.renard@onera.fr.

${ }^{\dagger}$ Chief senior research scientist, Aerodynamics, Aeroelasticity, Acoustics Department, Advanced Turbulence Modelling \& Simulation Unit.

${ }_{\ddagger}^{\ddagger}$ Senior research scientist, Aerodynamics, Aeroelasticity, Acoustics Department, Advanced Turbulence Modelling \& Simulation Unit. 


\section{WMLES of a supersonic turbulent boundary layer}

The first test case is a flat-plate zero-pressure-gradient turbulent boundary layer at an outer Mach number $M_{\infty}=2$. A wide range of Reynolds numbers is covered by the ZDES simulations, as illustrated by fig. 1. The mesh counts $7.7 \cdot 10^{6}$ grid points, much less than what would be needed for a wall-resolved LES (approximately $100 \cdot 10^{6}$ points). In a compressible context, the following definitions of the Reynolds numbers are adopted:

$$
\begin{gathered}
\theta=\int_{0}^{\infty} \frac{\langle\rho\rangle\langle u\rangle}{\rho_{\infty} U_{\infty}}\left(1-\frac{\langle u\rangle}{U_{\infty}}\right) d y \\
\operatorname{Re}_{\theta}=\frac{\theta U_{\infty} \rho_{\infty}}{\mu_{\infty}} \\
\operatorname{Re}_{\delta_{2}}=\frac{\theta U_{\infty} \rho_{\infty}}{\langle\mu\rangle_{w}}=F_{\theta} \operatorname{Re}_{\theta}=\frac{\mu_{\infty}}{\langle\mu\rangle_{w}} R e_{\theta} \\
\tau_{w}=\langle\mu\rangle \partial_{y}\langle u\rangle(y=0) \\
\tau_{w}=\langle\rho\rangle_{w} u_{\tau}^{2} \\
\operatorname{Re}_{\tau}=\frac{\delta u_{\tau}\langle\rho\rangle_{w}}{\langle\mu\rangle_{w}} \\
y^{+}=\frac{y u_{\tau}\langle\rho\rangle_{w}}{\langle\mu\rangle_{w}}
\end{gathered}
$$

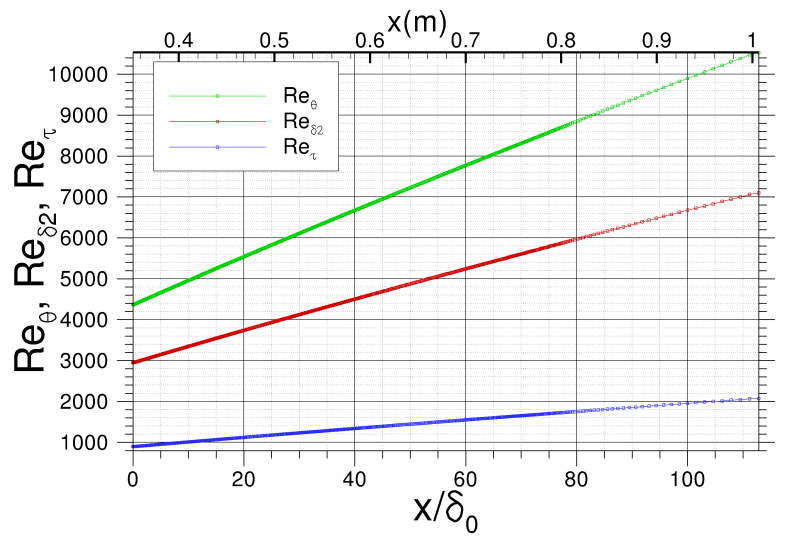

Fig. 1 Reynolds number range covered by the FP ZPG TBL case at $M_{\infty}=2$.

For the ZDES mode 3 computations, two different RANS/LES interface settings are considered, as introduced in [6]. The first interface is set at $d_{w}^{\text {interface }} / \delta=0.125$ based on the local boundary layer thickness. The second interface is located at $d_{w}^{\text {interface }+}=3.9 \sqrt{R e_{\tau}}$, i.e. close to the geometric center of the logarithmic layer.

The results are post-processed in order to enable a comparison with incompressible turbulent boundary layers following the findings of Hopkins \& Inouye [13]. Regarding the mean skin friction coefficient, it is first defined by:

$$
C_{f}=\frac{\tau_{w}}{1 / 2 \rho_{\infty} U_{\infty}^{2}}
$$

Then the mean skin friction $C_{f}$ is reduced to the incompressible case $C_{f i}$ for comparison using the Van Driest II approach [13]:

$$
\begin{gathered}
C_{f i}=F_{c} C_{f} \\
F_{c}=\left(\frac{\langle T\rangle_{w}}{T_{\infty}}-1\right)\left(\arcsin \left(\left(1-\frac{T_{\infty}}{\langle T\rangle_{w}}\right)^{1 / 2}\right)\right)^{-2}
\end{gathered}
$$


The relation $C_{f i}\left(R e_{\delta_{2}}\right)$ is almost independent of the Mach number ([13]). In addition, two boundary layers at different Mach numbers have relatively close $R e_{\tau}$ if they have the same $R e_{\delta_{2}}$ ([14]). For this reason, $C_{f i}$ and the turbulent profiles are compared with the incompressible case at the same $R e_{\delta_{2}}$ (or at the same $R e_{\tau}$ for quantities other than $C_{f i}$ ).

The prediction of mean skin friction illustrated in fig. 2] and compared with incompressible data after rescaling is quite satisfying. After the relaxation following the inflow condition, the ZDES mode 3 WMLES technique provides an accuracy better than $5 \%$ with respect to the Coles-Ferhnolz correlation calibrated by [15] for both RANS/LES interface height settings. This result is consistent with the previously reported behavior of ZDES mode 3 at low Mach numbers, indicating that the transposition of the method into supersonic conditions seems successful.

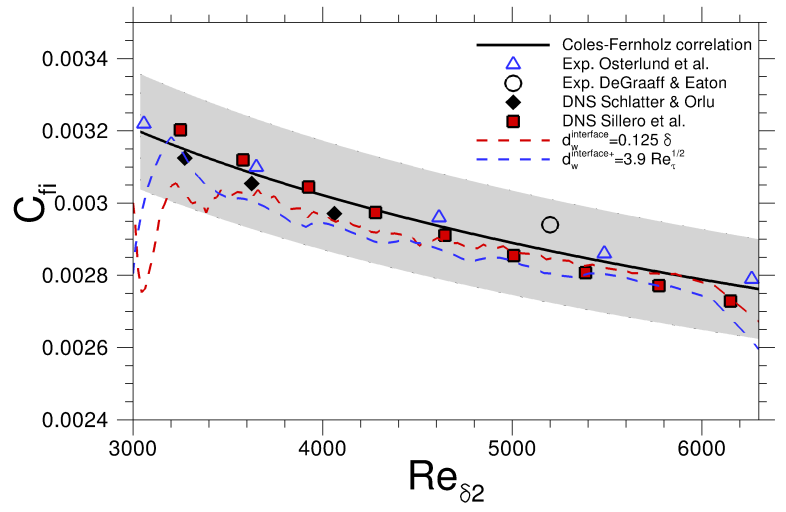

Fig. 2 Mean skin friction predicted by the ZDES simulations of the flat plate zero-pressure gradient turbulent boundary layer at $M_{\infty}=2$ rescaled for comparison with incompressible flows following [13].

This is further confirmed by the turbulent profiles compared with experimental / DNS data at close values of $R e_{\delta_{2}}$, with the mean velocity profile in fig. 3 and the Reynolds stresses in fig. 4 and 5 . Here the mean velocity has been Van Driest transformed according to:

$$
\langle u\rangle^{*}=\int_{0}^{\langle u\rangle} \sqrt{\frac{\langle\rho\rangle}{\langle\rho\rangle_{w}}} d u
$$

as well as the Reynolds stresses:

$$
\begin{gathered}
R_{i j}=\left\langle u_{i}^{\prime} u_{j}^{\prime}\right\rangle \\
R_{i j}^{*}=\frac{\langle\rho\rangle}{\langle\rho\rangle_{w}} R_{i j}
\end{gathered}
$$

Note that $\delta$ is still defined by $\langle u\rangle(y=\delta)=0.99 U_{\infty}$. The results confirm that the behaviour of the ZDES mode 3 technique at $M_{\infty}=2$ is very similar to its low Mach-number performance. It should be emphasized that most of the outer layer dynamics is resolved rather than modelled, as shown in fig. 4 making it a true WMLES approach. Similarly to the low Mach number results, the mean velocity profile somewhat deviates from the logarithmic law near the RANS/LES interface. The streamwise turbulent intensity is slightly underpredicted in the middle of the outer layer while it is overpredicted just below the RANS/LES interface when the latter is set at $d_{w}^{\text {interface }}+=3.9 \sqrt{R e_{\tau}}$. These results confirm that the outer-scaled interface setting $\left(d_{w}^{\text {interface }} / \delta=0.125\right.$ here $)$ is a good compromise for ZDES mode 3 .

\section{WMLES of a shock-wave boundary layer interaction}

The second test case is designed to investigate the capacity of the ZDES mode 3 technique to simulate the interaction of a supersonic $M_{\infty}=2$ turbulent boundary layer with an oblique shock wave. The previous case of a flat-plate zero pressure gradient turbulent boundary layer is retained but an incident shock wave producing a deviation angle of $8^{\circ}$ is added. The interaction features a mild flow separation near the shock foot, as illustrated by a RANS SA [9] simulation (fig. 6). It occurs at a Reynolds number $R e_{\delta_{2}}=5860$ which is higher than what could be easily achieved by DNS or WRLES (the latter would have required an estimated $100 \cdot 10^{6}$ grid points), demonstrating the interest of WMLES in this hybrid RANS/LES context where the mesh counts only $7.7 \cdot 10^{6}$ points. The interface setting $d_{w}^{\text {interface }} / \delta=0.125$ is 


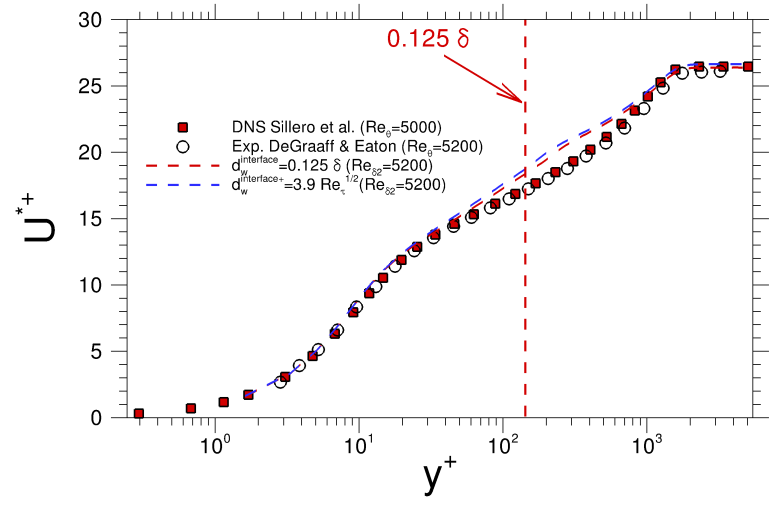

Fig. 3 Mean velocity profile predicted by the ZDES simulations of the FP ZPG TBL at $M_{\infty}=2$ rescaled for comparison with incompressible flows following [13].

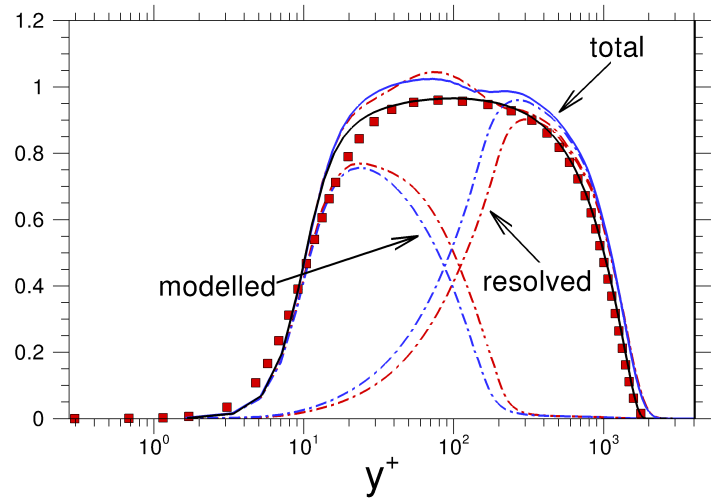

Fig. 4 Reynolds shear stress profiles predicted by the ZDES simulations of the FP ZPG TBL at $M_{\infty}=2$ rescaled for comparison with incompressible flows following [13].

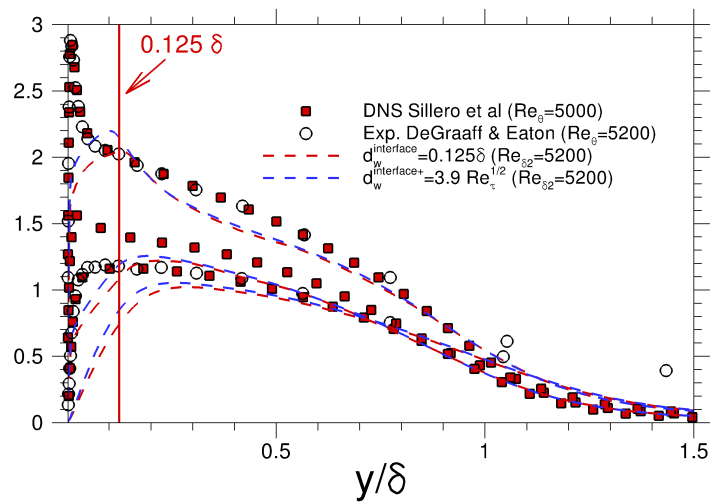

Fig. 5 Normal Reynolds stress profiles predicted by the ZDES simulations of the FP ZPG TBL at $M_{\infty}=2$ rescaled for comparison with incompressible flows following [13].

retained for the simulation. The fluctuations resolved by ZDES mode 3 are illustrated in fig. 7, 8 and 9 . The eddy viscosity field depicted in fig. 8 reveals that the outer layer is almost entirely resolved rather than modeled.

The interaction region is further described in figure 10 . The mean wall pressure evolution reveals the smoothing of 

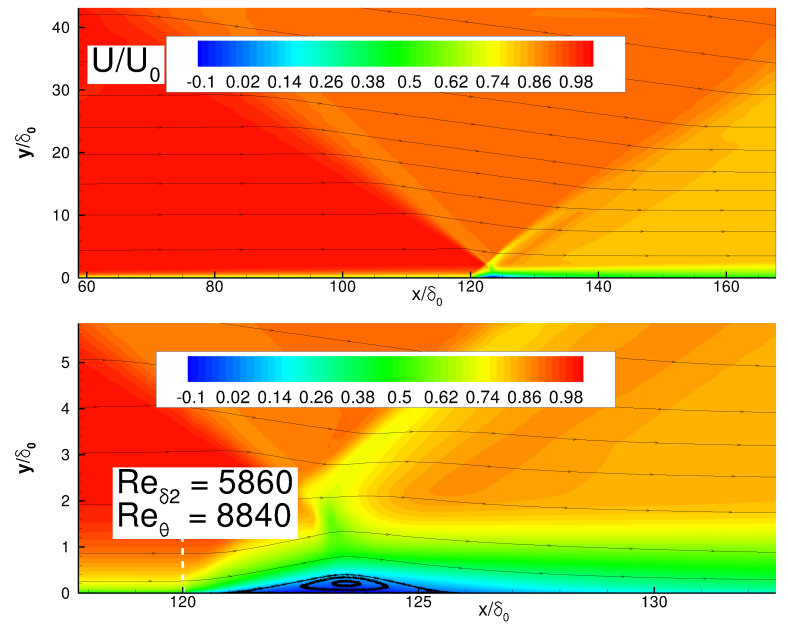

Fig. 6 RANS SA simulation of the shock-wave boundary layer interaction case.

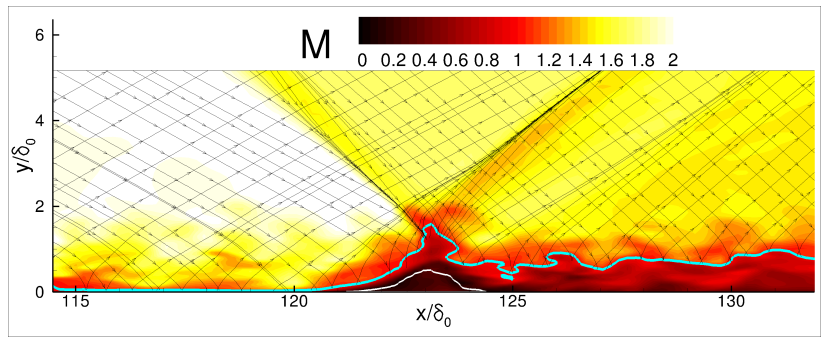

Fig. 7 Characteristic lines in the ZDES simulation of the shock-wave boundary layer interaction, together with the sonic line (in blue) and the $u=0$ instantaneous streamwise velocity isoline (in white).

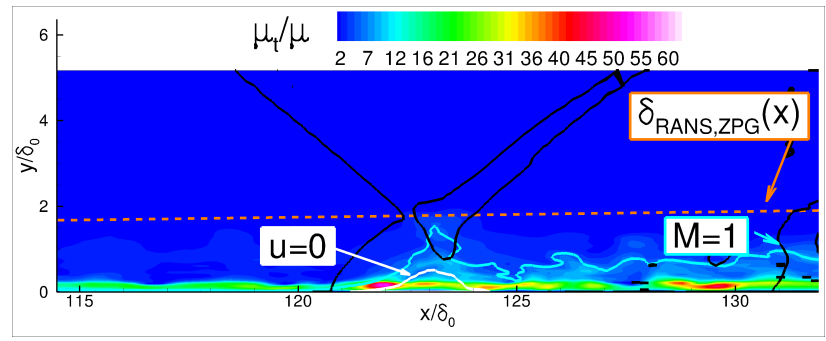

Fig. 8 Eddy viscosity field in the ZDES simulation of the shock-wave boundary layer interaction, together with pressure iso-surfaces.

the average pressure jump at the foot of the shock wave by the unsteadiness resolved in ZDES compared with the steady RANS simulation. This gradual pressure increase is a feature of the experimental data in a slightly different case (IUSTI experiment [16] with an upstream flow at $M_{\infty}=2.3$ and a deviation of $8^{\circ}$ ). It should be noted that the interaction in the IUSTI experiment is at a lower Reynolds number $\left(\operatorname{Re}_{\delta_{2}}\right.$ of the order of 3000$)$. The non-dimensionalised pressure jump $\Delta P /\left(2 \tau_{w}\right)$ is however very close in both cases (around 48 in the IUSTI case as well as in the present case). Remarkably, the resolved streamwise turbulent intensity obtained using ZDES in the present test case is qualitatively similar to the experimental results of the IUSTI case (fig. 10p, especially emphasizing the proper resolution of turbulent fluctuations in the shear layer associated to the separation bubble. Overall, the ZDES technique seems mature enough for the prediction of shallow flow separation at the shock foot, even though a more thorough analysis of the results with comparison with reference data in the exact same conditions would be needed to gain even more confidence in the technique. It should be emphasized that the essential unsteady phenomena are obviously captured at a computational cost more than ten times as small as for a WRLES, and this saving ratio would be even larger at higher Reynolds numbers. 


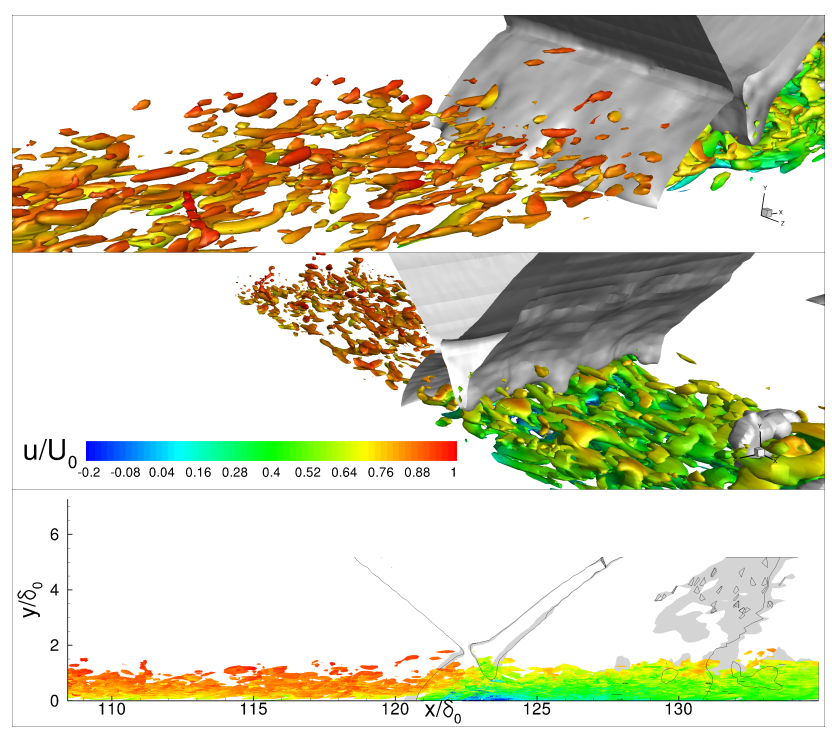

Fig. 9 Isosurface of the Q criterion in the ZDES simulation of the shock-wave boundary layer interaction, together with pressure iso-surfaces.
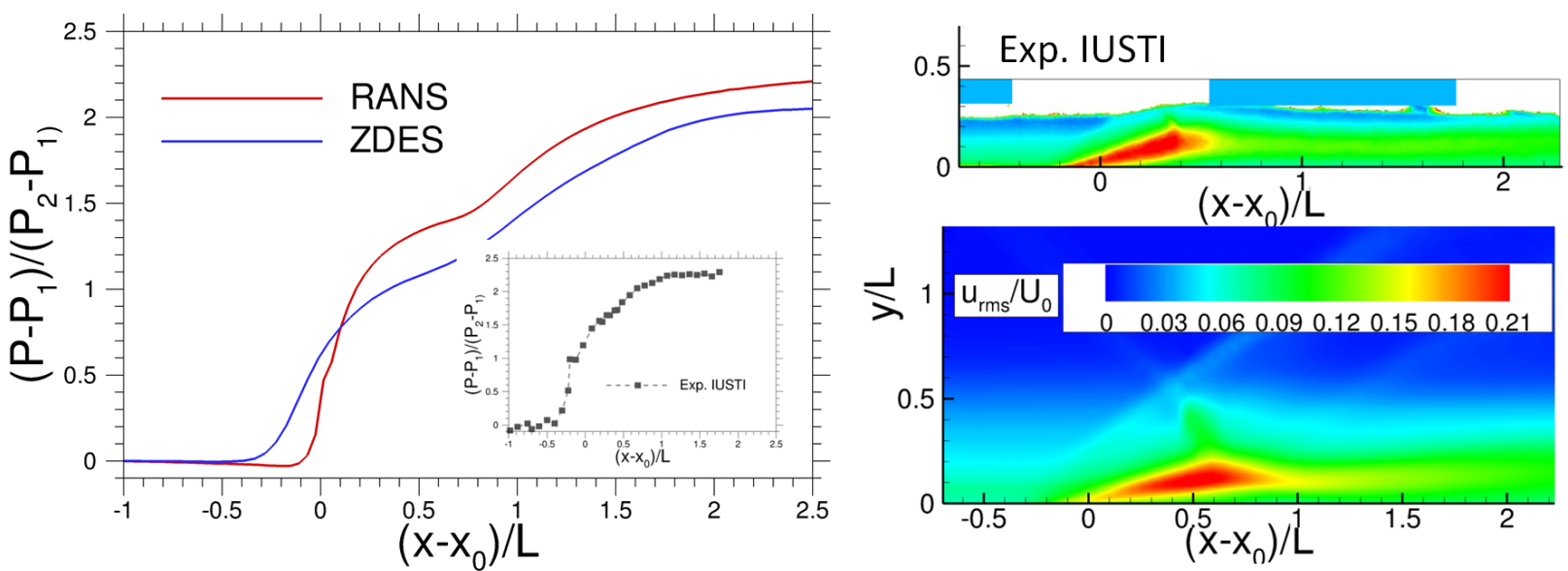

Fig. 10 Comparison of the interaction region: RANS, ZDES and IUSTI experiment [16], non-dimensionalized by the interaction length $L=x_{\text {imp }}-x_{0}$ with the mean separation location $x_{0}$ and the incident shock impingement location $x_{\text {imp. }}$

\section{Low-noise turbulent inflow for compressible WMLES}

For WMLES of fully turbulent boundary layers, resolved turbulence must be injected into the flow. This means that the ZDES mode 3 technique is used in conjunction with a turbulent injection method. However, in a compressible framework, the acoustic footprint of this injection may be problematic, especially for aeroacoustic studies. The last test case is consequently designed for the study of the acoustic footprint of the resolved turbulence generation needed for WMLES. A low Mach number $\left(M_{\infty}=0.21\right)$ flat-plate zero-pressure-gradient turbulent boundary layer case is simulated by the ZDES mode 3 technique in WMLES mode with the RANS/LES interface set at $d_{w}^{\text {interface }} / \delta=0.125$.

The new approach of resolved turbulence generation with a low-noise signature recently introduced by Deck et al. [1] is compared with more classical approaches, namely based on the Synthetic Eddy Method [17] and on White Noise injection [11, 18, 19]. The new approach relies on roughness elements described as immersed boundaries (ZIBC, Zonal Immersed Boundary Conditions as described in [20]) whose length scales are selected in order to disturb the boundary layer profile and generate large scale vorticity. The fluctuations are then enhanced by a dynamic forcing approach (inspired by Spille-Kohoff \& Kaltenbach and adapted to ZDES by [11, 19]), enabling a quick transition from 
the vorticity generated by the roughness to fully developed realistic turbulence as illustrated in fig. 11 The parameters of the different approaches are summarized in fig. 12

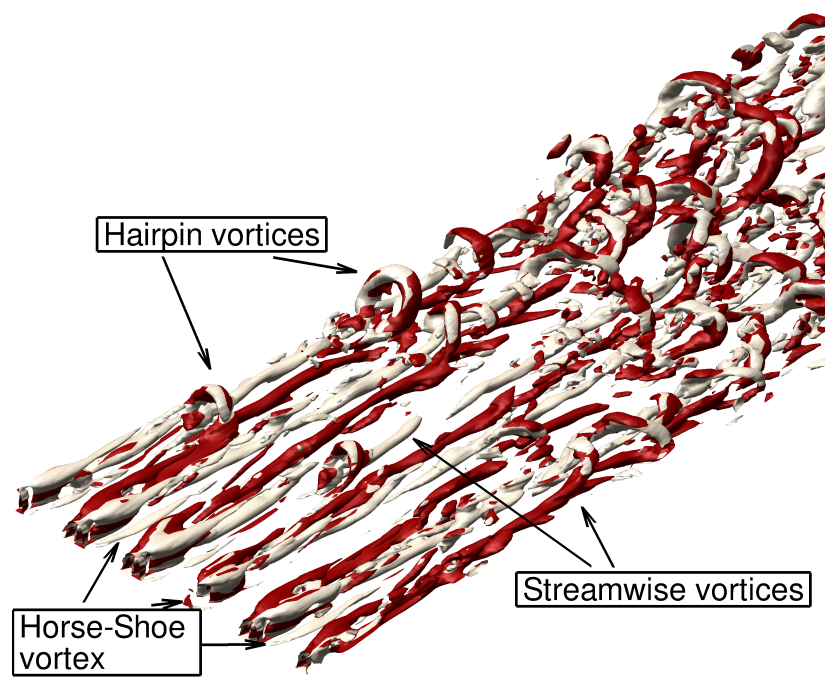

Fig. 11 Iso-surface of the $Q$ criterion coloured by the streamwise vorticity, near the inflow of the FP ZPG TBL case with the new resolved turbulence generation approach.

\begin{tabular}{|c|c|c|c|c|c|c|}
\hline Case & A1 & \multicolumn{2}{|l|}{ A2 } & \multicolumn{2}{|l|}{ B } & C1 to $\mathrm{C} 4$ \\
\hline $\begin{array}{l}\text { Fluctuations } \\
\text { generation }\end{array}$ & $\begin{array}{l}\text { Synthetic Eddy } \\
\text { Method }\end{array}$ & $\begin{array}{l}\text { Synthetic } \\
\text { Method }\end{array}$ & Eddy & White Noise & & $\begin{array}{l}\text { ZIBC roughness } \\
\text { elements }\end{array}$ \\
\hline Dynamic Forcing & No & Yes & & Yes & & Yes \\
\hline & & Case & C1 & $\mathrm{C}_{2}$ & $\mathrm{C} 3$ & $\mathrm{C}_{4}$ \\
\hline & & $\lambda_{z} / \delta_{0}$ & 1 & 1 & 0.5 & 0.5 \\
\hline & & $h / \delta_{0}$ & 0.6 & 0.3 & 0.3 & 0.6 \\
\hline
\end{tabular}

Fig. 12 Parameters of the resolved turbulence injection approaches.

Using the new approach, the mean skin friction relaxation distance is as short as with more common turbulent inflow conditions (fig. (13) as it can be as low as $7 \delta_{0}$. Besides, satisfying velocity profiles are obtained (fig. 14), the only visible shortcomings being related to ZDES mode 3 as a WMLES technique as already described earlier in this paper, but not to the injection approach.

In comparison with the strong spurious acoustic footprint of turbulence injection by the classical synthetic eddy method [21] illustrated in fig. 15, the new approach significantly reduces the spurious noise (fig. 16]. This is quantified by a comparison of the pressure levels in fig. 17 and 18 . Comparing the profile of rms pressure with DNS data especially suggests that only the computations made with the new injection approach are not excessively polluted by spurious noise. Of interest, it can be seen that the pressure fluctuations do penetrate through the inner RANS layer down to the wall, which is coherent with the penetration of Reynolds stresses already observed in previous studies, e.g. [5, 6]. For this reason, the wall pressure signals are further investigated in the following.

The spurious nature of the wall pressure field when classical turbulent inflow conditions are used is confirmed by spectral analysis, whereas the power spectral density obtained with the new approach shows no spurious peaks (fig. 19). The present results and their extrapolation to higher Reynolds numbers based on predictions by [22] suggest that at high Reynolds number, approximately $50 \%$ of the wall pressure variance may be resolved by a technique such as ZDES mode 3 , corresponding to the lower-half of the spectral frequency range. Given the significant reduction of computational cost compared with WRLES, this makes ZDES mode 3 a technique of interest for the prediction of low-frequency wall pressure fluctuations. The physical relevance of the wall pressure signals obtained with ZDES and the new injection 


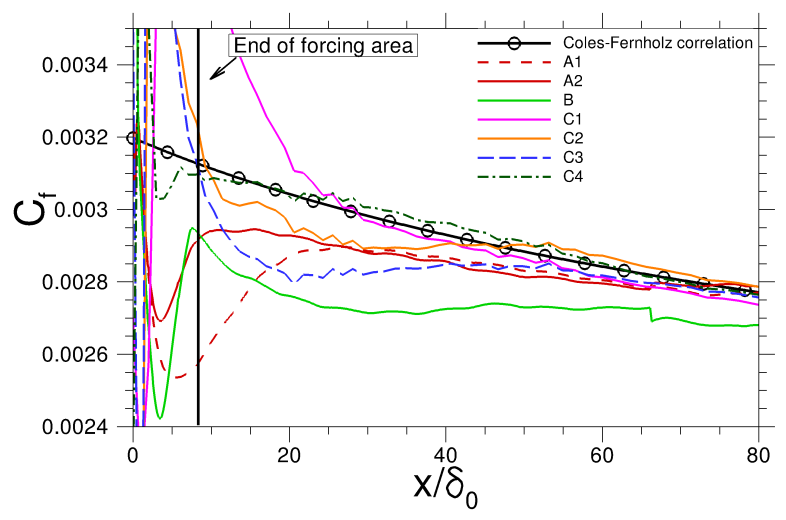

Fig. 13 Mean skin friction evolution near the inlet with different resolved turbulence generation methods: A, synthetic eddy method; $B$, white noise; $C$, new approach.

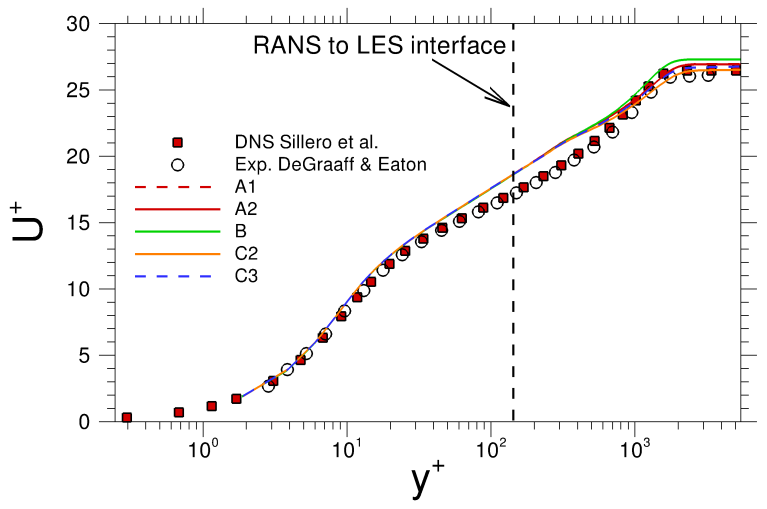

Fig. 14 Mean velocity profile at $R e_{\theta}=5200$ obtained with different resolved turbulence generation methods: $A$, synthetic eddy method; $B$, white noise; $C$, new approach.

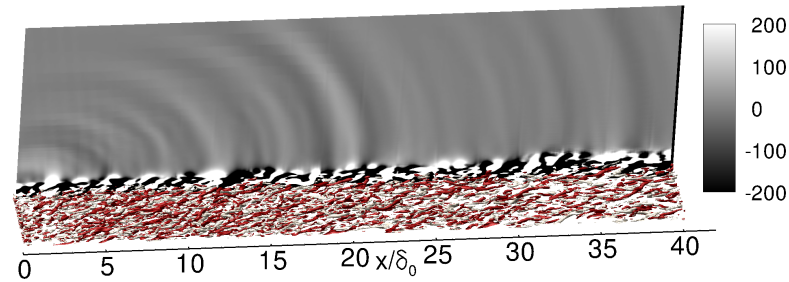

Fig. 15 Iso-surface of the $\mathbf{Q}$ criterion coloured by the streamwise vorticity and instantaneous field of $-\partial \rho / \partial t$ (gray scale) with a Synthetic Eddy Method inflow.

approach is further confirmed in fig. 20, where the intermittency of the wall signal, related to the intermittent nature of turbulence, is revealed by the higher tails of its probability density function compared with a Gaussian behavior (which corresponds to the spurious noise cases where the intermittent physical signal is masked by the spurious signal).

From a theoretical point of view, using the new turbulence injection approach in a supersonic boundary layer does not require any a priori modification. This has to be verified in a new test case which is out of the scope of the present study. 


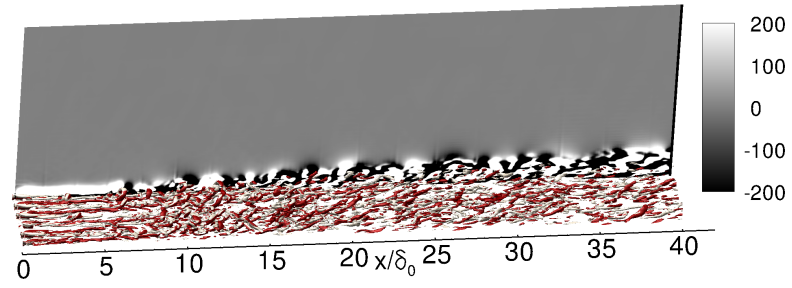

Fig. 16 Iso-surface of the $\mathbf{Q}$ criterion coloured by the streamwise vorticity and instantaneous field of $-\partial \rho / \partial t$ (gray scale) with the new resolved turbulence generation approach.

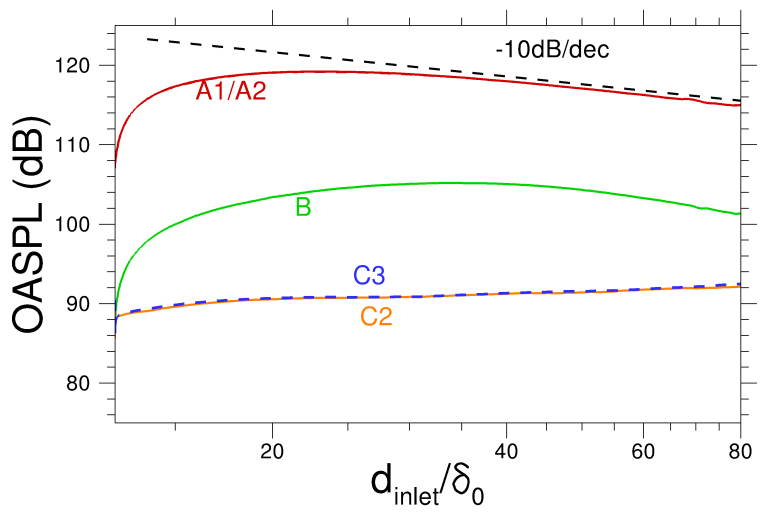

Fig. 17 Streamwise evolution of $O A S P L=20 \log _{10}\left(\frac{P_{\text {rms }}}{2 \cdot 10^{-5} \mathbf{P a}}\right)$ with the distance to the inlet $d_{\text {inlet }} / \delta_{0}$ at constant altitude $y=12.5 \delta_{0}$ with different resolved turbulence generation methods: A, synthetic eddy method; $\mathbf{B}$, white noise; $C$, new approach.

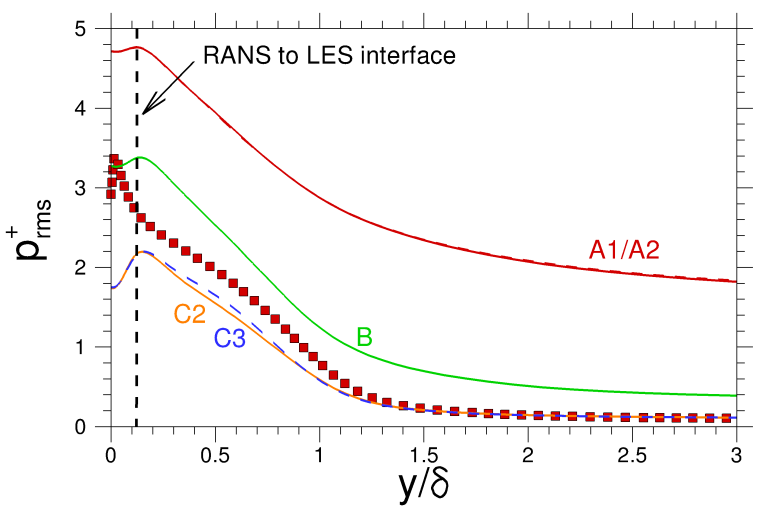

Fig. 18 Wall-normal distribution of pressure rms at $R e_{\theta}=5200$ with different resolved turbulence generation methods: A, synthetic eddy method; $B$, white noise; $C$, new approach.

\section{Conclusion}

The present study has demonstrated the use of the ZDES technique for WMLES studies using its mode 3 in a compressible context. Firstly, the performance in supersonic boundary layers has been shown to be equivalent to the previous results obtained at low Mach numbers. Secondly, this paves the way towards the simulation of complex shock wave / boundary layer interactions, as illustrated by an example at Mach 2 where the most salient features of the unsteadiness appear to be properly captured at a relatively low computational cost. Finally, a new resolved turbulence injection approach introduced by [1] is compared with more classical approaches, revealing in a low Mach number test case that the new approach strongly mitigates the spurious acoustic footprint usually induced by classical methods. The 


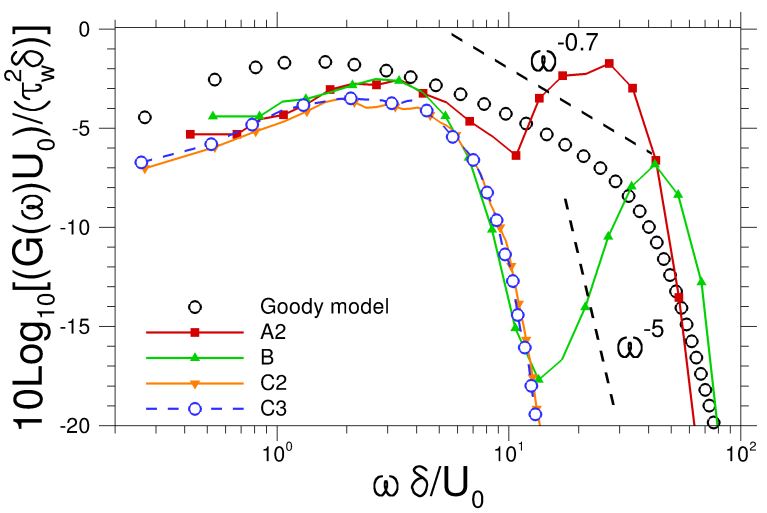

Fig. 19 Power Spectral Density of wall pressure fluctuations at $R e_{\theta}=5200$ with different resolved turbulence generation methods: A, synthetic eddy method; $B$, white noise; $C$, new approach.

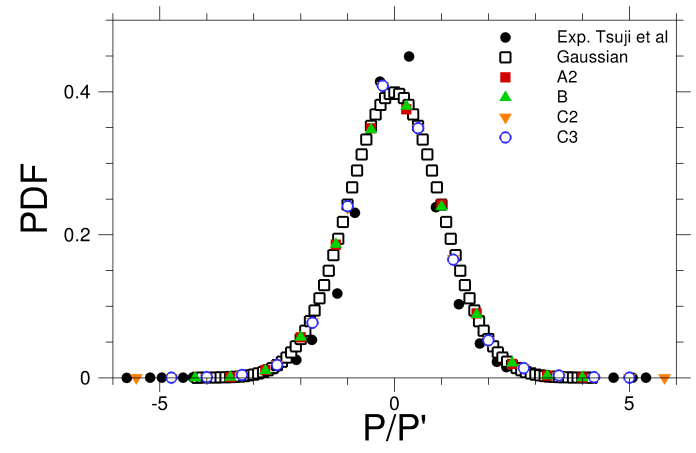

(a) linear scales

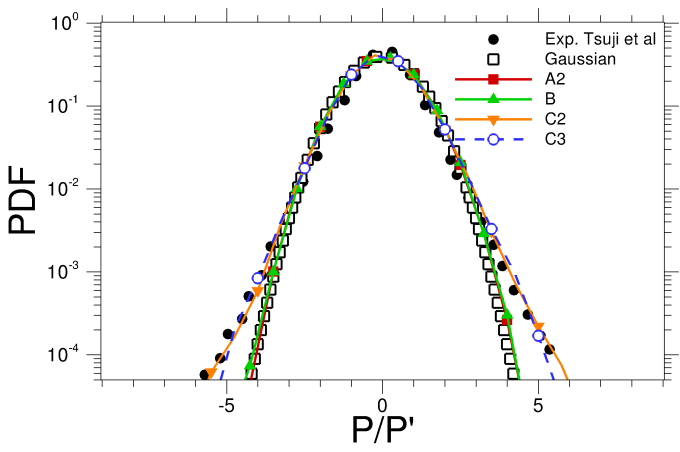

(b) semilogarithmic scales

Fig. 20 PDF of wall pressure fluctuations normalized by their root mean squared value at $\operatorname{Re}_{\theta}=5200$.

analysis of the wall pressure signals suggests that ZDES mode 3 may be used together with the new injection strategy for the prediction of the low-frequency range of the spectrum corresponding to $50 \%$ of the wall pressure variance at high Reynolds number.

Future work should involve the use of the new injection approach in supersonic flows. Overall, this study suggests the maturity of the ZDES mode 3 technique for compressible flows with both supersonic and aeroacoustic problems being within reach of the method.

\section{Acknowledgments}

The authors wish to thank all the people involved in the past and present evolution of the FLU3M code. The ZIBC approach has been developed in the framework of the research project ALLIGATOR funded by ONERA. Parts of this work are also related to the EU collaborative research project Go4Hybrid, funded by the European Community in the 7th Framework Programme, under Contract No. 605361, and to the research project OPOSSUM funded by ONERA.

\section{References}

[1] Deck, S., Weiss, P.-E., and Renard, N., "A rapid and low noise switch from RANS to WMLES on curvilinear grids with compressible flow solvers," Journal of Computational Physics, Vol. 363, 2018, pp. 231-255.

[2] Deck, S., "Recent improvements in the Zonal Detached Eddy Simulation (ZDES) formulation," Theoretical and Computational Fluid Dynamics, Vol. 26, 2012, pp. 523-550. 
[3] Deck, S., Gand, F., Brunet, V., and Khelil, S. B., "High-fidelity simulations of unsteady civil aircraft aerodynamics: stakes and perspectives. Application of Zonal Detached Eddy Simulation (ZDES)," Philosophical Transactions of the Royal Society A, Vol. 372,2014, p. 20130325.

[4] Deck, S., Weiss, P.-E., Pamiès, M., and Garnier, E., "Zonal Detached Eddy Simulation of a spatially developing flat plate turbulent boundary layer," Computers \& Fluids, Vol. 48, 2011, pp. 1-15.

[5] Deck, S., Renard, N., Laraufie, R., and Sagaut, P., "Zonal Detached Eddy Simulation (ZDES) of a spatially developing flat plate turbulent boundary layer over the Reynolds number range $3150 \leq R e_{\theta} \leq 14$ 000," Physics of Fluids, Vol. 26, 2014 , p. 025116.

[6] Renard, N., and Deck, S., "Improvements in Zonal Detached Eddy Simulation for Wall Modeled Large Eddy Simulation," AIAA Journal, Vol. 53, No. 11, 2015, pp. 3499-3504. doi:10.2514/1.J054143.

[7] Shur, M. L., Spalart, P. R., Strelets, M. K., and Travin, A. K., "A hybrid RANS-LES approach with delayed-DES and wall-modelled LES capabilities,” International Journal of Heat and Fluid Flow, Vol. 29, 2008, pp. 1638-1649.

[8] Choi, J.-I., Edwards, J. R., and Baurle, R. A., "Compressible Boundary-Layer Predictions at High Reynolds Number Using Hybrid LES/RANS Methods,” AIAA Journal, Vol. 47 No 9, 2009, pp. 2179-2193.

[9] Spalart, P. R., and Allmaras, S. R., "A One-Equation Turbulence Model for Aerodynamic Flows," La Recherche Aérospatiale, , No. 1, 1994, pp. 5-21.

[10] Deck, S., Renard, N., Laraufie, R., and Weiss, P.-E., "Large scale contribution to mean wall shear stress in high Reynolds number flat plate boundary layers up to $R e_{\theta}=13$ 650," Journal of Fluid Mechanics, Vol. 743, 2014, pp. $202-248$. doi:doi:10.1017/jfm.2013.629.

[11] Laraufie, R., Deck, S., and Sagaut, P., "A dynamic forcing method for unsteady turbulent inflow conditions," Journal of Computational Physics, Vol. 230, 2011, pp. 8647-8663.

[12] Laraufie, R., and Deck, S., "Assessment of Reynolds stresses tensor reconstruction methods for synthetic turbulent inflow conditions. Application to hybrid RANS/LES methods," International Journal of Heat and Fluid Flow, Vol. 42, 2013 , pp. 68-78.

[13] Hopkins, E. J., and Inouye, M., "An evaluation of theories for predicting turbulent skin friction and heat transfer on flat plates at supersonic and hypersonic Mach numbers," AIAA Journal, Vol. 9, No. 6, 1971, pp. 993-1003.

[14] Pirozzoli, S., and Bernardini, M., "Turbulence in supersonic boundary layers at moderate Reynolds number," Journal of Fluid Mechanics, Vol. 688, 2011, pp. 120-168.

[15] Nagib, H. M., Chauhan, K. A., and Monkewitz, P. A., "Approach to an asymptotic state for zero pressure gradient turbulent boundary layers," Philosophical Transactions of the Royal Society A, Vol. 365, 2007, pp. 755-770.

[16] Piponniau, S., Dussauge, J. P., Debiève, J. F., and Dupont, P., "A simple model for low-frequency unsteadiness in shock-induced separation," Journal of Fluid Mechanics, Vol. 629, 2009, pp. 87-108.

[17] Pamiès, M., "Contrôle d'une chouche limite turbulente au moyen d'un micro-système distribué,” Ph.D. thesis, Université de Lille 1, 2008.

[18] Laraufie, R., Deck, S., and Sagaut, P., "A Rapid switch from RANS to WMLES for spatially developing boundary layers," Progress in Hybrid RANS-LES Modelling, NNFM117, edited by S. Fu W. Haase S.-H. Peng and D. Schwamborn, Springer-Verlag, Berlin Heidelberg, 2012, pp. 147-156.

[19] Deck, S., and Laraufie, R., "Numerical investigation of the flow dynamics past a three-element aerofoil," Journal of Fluid Mechanics, Vol. 732, 2013, pp. 401-444.

[20] Weiss, P., and Deck, S., "On the coupling of a zonal body-fitted/immersed boundary method with ZDES: application to the interactions on a realistic space launcher afterbody flow," Computers \& Fluids, in press, doi:10.1016/j.compfluid.2017.06.015, 2017. doi:https://doi.org/10.1016/j.compfluid.2017.06.015.

[21] Pamiès, M., Weiss, P.-E., Garnier, E., Deck, S., and Sagaut, P., "Generation of synthetic turbulent inflow data for large eddy simulation of spatially evolving wall-bounded flows," Physics of Fluids, Vol. 21, 2009, p. 045103.

[22] Aupoix, B., "Extension of Lysak's Approach to Evaluate the Wall Pressure Spectrum for Boundary Layer Flows," Flow Turbulence and Combustion, vol 94, No. 1, pp 63-78, 2015. 